\title{
Geneviève MASSARD-GUILBAUD, Histoire de la pollution industrielle. France (1789-1914)
}

\section{Guy Lemarchand}

\section{(e) OpenEdition \\ Journals}

Édition électronique

URL : https://journals.openedition.org/ahrf/12194

DOI : 10.4000/ahrf.12194

ISSN : 1952-403X

Éditeur :

Armand Colin, Société des études robespierristes

Édition imprimée

Date de publication : 1 septembre 2011

Pagination : 229-231

ISBN : 978-2-200-92700-4

ISSN : 0003-4436

Référence électronique

Guy Lemarchand, "Geneviève mASSARD-GUILBAUD, Histoire de la pollution industrielle. France (1789-1974)», Annales historiques de la Révolution française [En ligne], 365 | Juillet-septembre 2011, mis en ligne le 13 décembre 2011, consulté le 22 avril 2022. URL : http://journals.openedition.org/ahrf/12194; DOI : https://doi.org/10.4000/ahrf.12194

Ce document a été généré automatiquement le 22 avril 2022.

Tous droits réservés 


\title{
Geneviève MASSARD-GUILBAUD, Histoire de la pollution industrielle. France (1789-1914)
}

\author{
Guy Lemarchand
}

\section{RÉFÉRENCE}

Geneviève MASSARD-GUILBAUD, Histoire de la pollution industrielle. France (1789-1914), Paris, éd. de l'EHESS, 2010, 404 p., IBSN : 978-2-7132-2237-5, $27 €$.

1 Suivant le progrès des sciences sociales, la pollution et corrélativement l'environnement sont entrés depuis une vingtaine d'années dans le champ de la discipline historique et la crise actuelle du capitalisme n'a fait que renforcer l'importance de cette thématique. Au XIX ${ }^{\mathrm{e}}$ siècle et même jusqu'à 1980, les historiens n'ont pratiquement accordé aucune attention à l'un des principaux facteurs de la pollution, l'industrie, alors qu'ils consacraient des études de plus en plus nombreuses et sophistiquées sur le plan économique à celle-ci. À cet égard ce livre est novateur et d'autant plus utile que l'auteur a conçu le concept

de base de façon large. Il s'agit des nuisances portées par l'homme non seulement à la nature végétale, animale et géologique, mais également à la société et particulièrement dans la ville où s'accumule l'activité manufacturière depuis la révolution industrielle des $\mathrm{XVIII}^{\mathrm{e}}$-XIX ${ }^{\mathrm{e}}$ siècles. Ne pouvant évidemment tout traiter, Geneviève MassardGuilbaud a exclu de sa recherche la pollution provoquée par l'organisme urbain luimême, et Paris pour lequel quelques travaux sont déjà parus, ce qui lui laisse tout de même la vaste province. Elle a dépouillé les dossiers sur les établissements dangereux ou insalubres conservés dans les Archives Départementales, surtout pour Nantes, Clermont-Ferrand et Lyon, les débats parlementaires, les traités et rapports ponctuels scientifiques sur la chimie et l'hygiène publique ainsi que les articles des revues spécialisées comme les Annales des Mines ou la Revue pratique d'hygiène municipale, soit 
plus de 400 titres, outre la bibliographie historique de plus de 600 titres, littérature austère parfaitement maîtrisée.

Déjà, en face des nuisances l'Ancien Régime n'était pas resté inerte. Il s'en était même préoccupé plus qu'on ne pourrait le croire, poussé par les plaintes et procès intentés par les riverains des ateliers aux émanations et rejets infects. À la fin du XVIII ${ }^{\mathrm{e}}$ siècle la Société Royale de Médecine s'inquiète des dangers sanitaires suscités par les nouvelles fabrications chimiques. Mais si ponctuellement se multiplient les ordonnances d'intendants et les arrêts de Parlements mobilisés essentiellement sur la question des débris et déjections et celle des fumées, la solution préconisée se limite à l'éloignement de la nuisance à la périphérie de la ville et aucune législation d'ensemble n'est élaborée. La Révolution bouleverse la perspective, et, confirmée et amplifiée par l'Empire, assure en ce domaine le triomphe du libéralisme et des «intérêts ». Toutefois ce libéralisme entendez la priorité laissée à l'initiative des entrepreneurs - est soutenu et théoriquement encadré par la puissance publique, loin d'être débridé et anarchique.

En décembre 1789 il est décidé que les anciens règlements sont maintenus mais placés sous la surveillance des municipalités. Sous le Consulat, avec Chaptal, chimiste, propriétaire d'une fabrique d'acide sulfurique et ministre de l'Intérieur, au nom des avantages pour la nation de la croissance d'une industrie moderne, se noue une alliance tacite entre l'administration centrale soucieuse de réaliser le bon ordre prospère et d'établir une fiscalité fructueuse, les savants désireux de valoriser leurs découvertes et les capitalistes à la recherche d'occasions de profits conséquents, accord dont, j'ajouterai, on retrouve maints exemples jusqu'au xxI ${ }^{e}$ siècle. Le but de cette entente, qui va se prolonger effectivement bien au-delà de 1815 , est de réduire l'« intolérable arbitraire » qui freinerait l'essor des nouveautés bienfaisantes. En sortira le décret du 15 octobre 1810 qui règle le problème jusqu'à la première guerre mondiale. Le texte reprend les propositions de l'Académie des Sciences de 1804 et il considère comme nuisances seules les odeurs incommodes ou éventuellement insalubres. Il classe les industries en trois catégories : celles qui doivent être éloignées des habitations et qui sont soumises à autorisation du Conseil d'État, celles qui peuvent être installées librement mais moyennant enquête du maire et accord du préfet, celles qui sont dispensées de toute inspection mais doivent tout de même avoir l'agrément du souspréfet. Quand un établissement aura rempli positivement les formalités requises, nul ne pourra réclamer son départ sans jugement du Conseil d'État. Donc l'État se montre interventionniste puisqu'il contrôle la validité de la novation, mais son accord vaut protection durable, la justice civile étant déboutée de toutes plaintes de tiers. Le libéralisme n'est pas seulement une idée pure contemplée au fond de la caverne de Platon. Cela d'autant moins que les institutions qui auraient pu et dû surveiller et contrebattre l'influence des entrepreneurs sont largement pénétrées par eux. Le Comité Consultatif des Arts et Manufactures créé en 1791-1806, dont les membres sont nommés par le Chef de l'État, comporte de nombreux chimistes et industriels. Le Conseil d'État mis en place par la constitution de l'an VIII, dont l'auteur aurait pu rappeler le recrutement de juristes et de proches des milieux d'affaires, se fait laxiste et tend à remplacer l'éloignement obligatoire des établissements incriminés par des prescriptions techniques. Les corps des ingénieurs des Mines et des ingénieurs des Ponts, fréquemment consultés par les pouvoirs publics, sont partisans eux-mêmes du développement avant tout de l'industrie. Les maires, responsables par les lois municipales de 1790 et 1837 de la police sanitaire, ne peuvent aller contre l'autorité des 
préfets et sont souvent aussi industrialistes. En face, la Société Royale de Médecine, dissoute en 1794, ne réapparaît qu'en 1820, les Conseils départementaux d'hygiène créés peu à peu dans les grandes villes à partir de 1817, à l'image du Comité de salubrité de Paris institué en 1810, sont peu entendus, leurs avis étant consultatifs et pas toujours sollicités.

Le résultat est que les contraintes pesant sur les entreprises sont très limitées et même que, avec la bienveillance de l'administration, elles ne sont pas respectées, nombre d'usines étant bâties et se développant sans avoir demandé l'autorisation nécessaire. Les patrons refusent d'intégrer dans leurs comptes d'exploitation le coût des rejets et de l'assainissement. $\mathrm{Vu}$ leurs conditions de travail souvent difficiles, les ouvriers n'appliquent pas les précautions hygiénistes qui leur compliqueraient la tâche. Pourtant, avec l'expansion des activités à nuisance et avec l'urbanisation, une réaction timide s'esquisse à partir du milieu du XIX ${ }^{e}$ siècle, et davantage après 1870 . Sous forme de lettres individuelles ou envoyées stéréotypées en série, et de pétitions, les protestations des victimes s'accroissent. Sur le plan idéologique les notions de nuisance et de santé publique s'élargissent et se rapprochent l'une de l'autre. On en vient à considérer certaines fumées d'origine chimique comme dangereuses pour l'organisme humain et, tardivement, à inscrire le bruit dans les nuisances, alors que pendant longtemps on s'en était tenu à désigner comme négative la putréfaction des matières organiques végétales comme le rouissage du chanvre, ou animales comme les déchets des abattoirs. Ainsi les prescriptions inscrites dans les autorisations accordées aux entrepreneurs s'allongent au fil des décennies. Néanmoins le progrès demeure limité et n'est pas à la mesure de l'augmentation du nombre des usines et de la diversification de leur production. Deux données sont significatives à ce propos : la rareté des révisions de la nomenclature des établissements édictée en 1811 - trois seulement, en 1815, 1866, 1883, - et il y a également l'échec des projets de loi du ministère du Commerce étendant la répression des contraventions à la réglementation sur les mêmes établissements, devant la Chambre des députés en 1889, devant le Sénat en 1893.

Un tel résumé rend mal compte de la richesse de l'ouvrage qui aborde aussi bien les débuts de la chimie scientifique au $\mathrm{XVIII}^{\mathrm{e}}$ siècle et l'évolution des techniques au $\mathrm{XIX}^{\mathrm{e}}$ siècle, que le rôle de la spéculation immobilière dans la localisation des usines dans les quartiers d'habitation, ou que l'œuvre des conseils d'hygiène que l'auteur réhabilite partiellement. En outre pour chaque grande question Geneviève Massard n'analyse pas seulement le discours des acteurs mais précise les idées et les faits par l'utilisation de schémas simples et de photographies d'époque et par la présentation de cas concrets comme, par exemple, celui de l'entreprise Figueroa de fonte de plomb à Marseille en 1846-1853. On ne peut que souhaiter un livre de la même qualité pour la période 1914-2011. 